\title{
The novel long non-coding RNA PRNCR1-2 is involved in breast cancer cell proliferation, migration, invasion and cell cycle progression
}

\author{
DANMEI PANG ${ }^{1 *}$, QIAN HU ${ }^{1,2^{*}}$, XIAOSHAN LAN ${ }^{1}$, YINGXIN LIN $^{1}$, HAIBO DUAN $^{1}$, \\ SHUO CAO ${ }^{1}$, YAODONG LIN ${ }^{1}$, LITAO LI ${ }^{1}$, FENG PENG $^{1}$ and FENGTAO PAN ${ }^{1}$ \\ ${ }^{1}$ Department of Breast Cancer Oncology, Foshan Hospital of Sun Yat-Sen University, Foshan, Guangdong 528000; \\ ${ }^{2}$ Department of Oncology, Sun Yat-Sen University Cancer Center, Guangzhou, Guangdong 510060, P.R. China
}

Received May 8, 2018; Accepted November 22, 2018

DOI: $10.3892 / \mathrm{mmr} .2018 .9789$

\begin{abstract}
Long non-coding RNAs (lncRNAs) have recently been reported to act as important mediators of tumor initiation and progression. The present study aimed to investigate the expression and pathogenic roles of the IncRNA prostate cancer-associated non-coding RNA (PRNCR)1-2 in breast cancer. The expression levels of PRNCR1-2 were detected in breast cancer tissues and numerous breast cancer cell lines using reverse transcription-quantitative polymerase chain reaction. Depletion of PRNCR1-2 expression in breast cancer cells was conducted through small interfering RNA-mediated silencing. Subsequently, cell proliferation was assessed by MTS assay, cell migration and invasion capacities were evaluated using the Transwell culture system, and cell cycle progression and apoptosis were analyzed by flow cytometry. Protein expression levels of the signaling components checkpoint kinase 2 (CHK2), protein kinase B (AKT), phosphorylated (p)-CHK2 and p-AKT were measured by western blotting. The results demonstrated that PRNCR1-2 expression was significantly elevated in breast cancer tissues compared with in adjacent normal tissues. Furthermore, depletion of PRNCR1-2 in HS-578T and MDA-MB-231 breast cancer cells markedly suppressed their proliferation rates, migration and invasion capacities, and cell cycle progression; however, it had no effect on cell apoptosis. In addition, PRNCR1-2 depletion increased CHK2 phosphorylation and decreased AKT
\end{abstract}

Correspondence to: Dr Danmei Pang, Department of Breast Cancer Oncology, Foshan Hospital of Sun Yat-Sen University, 81 North Linnan Road, Chancheng, Foshan, Guangdong 528000, P.R. China

E-mail:pdmei@fsyyy.com

${ }^{*}$ Contributed equally

Key words: PRNCR1-2, long non-coding RNA, breast cancer, proliferation, migration and invasion, cell cycle progression phosphorylation in HS-578T and MDA-MB-231 cells. In conclusion, the lncRNA PRNCR1-2 may promote breast cancer cell proliferation, migration, invasion and cell cycle progression.

\section{Introduction}

Breast cancer is a major human malignancy and is the second leading cause of cancer-associated mortality in women worldwide, following lung cancer (1). As estimated by the American Cancer Society, there will be $>266,000$ new cases of breast cancer and $>40,000$ cases of breast cancer-associated mortality in the next 10 years in the United States, thus accounting for 30 and $14 \%$ of total cancer cases and cases of cancer-associated mortality, respectively (1). Survival rates of patients with breast cancer is relatively low, particularly in developing countries; however, a 5-year survival rate of $>80 \%$ has been achieved in England and the United States (2). As a complex heterogeneous malignancy, breast cancer remains a severe public health concern, due to ambiguities regarding the stepwise pathological processes from normal breast tissue to metastatic cancer tissue $(3,4)$. Previous studies have revealed that the pathogenesis of breast cancer is accompanied and driven by a series of successive mutations in genetic and epigenetic networks in breast cells, finally resulting in activation of various hallmarks, malignant transformation and metastasis $(5,6)$. In recent years, it has been suggested that noncoding RNAs (ncRNAs) are responsible for genetic and epigenetic dysregulation, which is associated with various developmental and pathological processes, including tumorigenesis $(7,8)$. However, the specific roles of ncRNAs in breast cancer remain to be investigated.

Long ncRNAs (lncRNAs) refer to ncRNA transcripts containing >200 nucleotides, which are involved in post-transcriptional regulation of gene expression by interfering with microRNA functioning (9). Through modulating gene expression at the transcriptional, post-transcriptional and epigenetic levels, IncRNAs have been reported to be associated with various physiological and pathological processes, including stem cell development, neurogenesis and oncogenesis $(8,9)$. In various types of human cancer, IncRNAs act as promoters and 
maintainers of cancer initiation and progression; therefore, they have been considered potential biomarkers for clinical diagnosis, and also as therapeutic targets for the development of novel treatments $(10,11)$. Based on functional studies using cellular and animal models, it has been reported that IncRNAs are involved in various cancer-associated cellular phenotypes, including cell death inhibition, activation of invasion, proliferation maintenance, dysregulated cellular energetics, genomic instability and evasion of growth suppressors (12). LncRNAs have also been identified as key regulators in breast cancer. For example, the lncRNA HOX transcript antisense RNA, which promotes cancer metastasis by modulating chromatin states, has been identified as a potential prognostic marker of cancer metastasis for estrogen receptor (ER)-positive breast cancer (13). Furthermore, the IncRNA urothelial cancer-associated 1 has been reported to enhance breast tumor growth through suppression of p27 protein levels via competitive inhibition (14). An accumulating number of lncRNAs have been demonstrated to be associated with breast cancer initiation and progression, thus suggesting that functional elucidation of key lncRNAs in breast cancer cells may provide valuable information for clinical screening and management. Prostate cancer-associated ncRNA 1 (PRNCR1) is closely associated with resistance of prostate cancer to castration (15), colorectal cancer cell proliferation and cell cycle progression (16), and progression of other types of cancer (17). However, to the best of our knowledge, the implications of PRNCR1 in breast cancer progression have not been previously addressed.

In the present study, the expression levels of PRNCR1 were detected in clinical tissues from patients with breast cancer, as well as in numerous breast cancer cell lines. Subsequently, PRNCR1 expression was knocked down in breast cancer cells and a functional analysis was conducted. The present study identified an association between PRNCR1 and breast cancer, and therefore provided a novel candidate for ncRNA-based breast cancer diagnosis and treatment. These findings may also result in future elucidation of the pathological mechanisms underlying breast cancer initiation and progression.

\section{Materials and methods}

Cancer tissues and cell lines. Breast cancer tissues and paired adjacent normal tissues were collected from 20 patients at the Department of Breast Surgery, Foshan First People's Hospital (Foshan, China) between June 2015 and July 2017. Breast cancer was confirmed by pathological diagnosis post-operation, and the clinicopathological data of patients are displayed in Table I. The experimental procedures were approved by the Ethics Committee of Foshan First People's Hospital, and written informed consent was obtained from each participant prior to surgery. The breast cancer cell lines HS-578T (cat. no. HTB-126), MCF-7 (cat. no. HTB-22), MDA-MB-468 (cat. no. HTB-132), MDA-MB-231 (cat. no. HTB-26) and BT-549 (cat. no. HTB-122), and the mammary epithelial cell line MCF10A (cat. no. CRL-10317) were obtained from the American Type Culture Collection (Manassas, VA, USA). Breast cancer cells and the mammary epithelial cell line were cultured in Dulbecco's modified Eagle's medium (DMEM; Gibco; Thermo Fisher Scientific, Inc., Waltham, MA, USA)
Table I. Clinicopathological data of patients with breast cancer.

\begin{tabular}{lr}
\hline Characteristic & Number \\
\hline Age (years) & 12 \\
$<55$ & 8 \\
$>55$ & \\
Sex & 0 \\
Male & 20 \\
Female & \\
Distant metastasis & 10 \\
Absent & 10 \\
Present & \\
Histological grade & 3 \\
1 & 11 \\
2 & 6 \\
3 & \\
Tumor size (cm) & 6 \\
$\leq 2$ & 13 \\
$2-5$ & 1 \\
$>5$ & \\
\hline
\end{tabular}

containing $10 \%$ fetal bovine serum (Biowest, Nuaillé, France) in a humidified atmosphere supplied with $5 \% \mathrm{CO}_{2}$ at $37^{\circ} \mathrm{C}$.

Reverse transcription-quantitative polymerase chain reaction $(R T-q P C R)$. Relative lncRNA expression levels were measured in total RNA samples extracted from breast cancer tissues and cell lines by RT-qPCR. Prior to RNA extraction, breast tissues were homogenized in liquid nitrogen and breast cell lines were collected by centrifugation at $800 \mathrm{x}$ g for $10 \mathrm{~min}$ at room temperature, followed by three washes with PBS. Total RNA samples were extracted using TRIzol ${ }^{\circledR}$ solution (Invitrogen; Thermo Fisher Scientific, Inc.), according to the manufacturer's protocol. RNA concentrations were determined by spectrophotometer (NanoDrop ${ }^{\mathrm{TM}}$ 1000; NanoDrop; Thermo Fisher Scientific, Inc., Wilmington, DE, USA). Subsequently, $2.0 \mu \mathrm{g}$ RNA was used for cDNA synthesis by Moloney Murine Leukemia Virus reverse transcriptase (Promega Corporation, Madison, WI, USA) according to the manufacturer's protocol, and PCR analysis was performed using the 2X EasyTaq PCR SuperMix kit (cat. no. AS111-03; Beijing Transgen Biotech Co., Ltd., Beijing, China) according to the manufacturer's protocol. The thermocycling conditions for qPCR were: An initial denaturation at $95^{\circ} \mathrm{C}$ for $120 \mathrm{sec}$, followed by 40 cycles of $95^{\circ} \mathrm{C}$ for $15 \mathrm{sec}$ and $60^{\circ} \mathrm{C}$ for $30 \mathrm{sec}$ for primer annealing and elongation, ending with a melting curve step of $65^{\circ} \mathrm{C}$ to $95^{\circ} \mathrm{C}$ with an increment of $0.5^{\circ} \mathrm{C} / 5 \mathrm{sec} . \beta$-actin was used as an internal standard for quantification. The relative expression levels of genes were calculated using the $2^{-\Delta \Delta \mathrm{Cq}}$ method (18). For statistical analysis, at least three biological and technical replicates were performed. The following primers were used for detection of PRNCR1-2 expression: Forward, 5-CCTTTC CCTCATGACCCAGT-3 and reverse, ATTGGTGTGAGG GGAGTCTG. $\beta$-Actin: Forward, CATGTACGTTGCTAT CCAGGC and reverse, CTCCTTAATGTCACGCACGAT. 
Cell transfection. For knockdown of PRNCR1-2 expression, breast cancer cells were digested with trypsin solution, seeded into 6-well plates and cultured overnight at $37^{\circ} \mathrm{C}$ in an atmosphere containing $5 \% \mathrm{CO}_{2}$. Once cells reached $80 \%$ confluence, they were washed twice with PBS and added to $1.5 \mathrm{ml}$ fresh basal DMEM. Subsequently, the cells were mixed with $10 \mu \mathrm{l}$ $20 \mu \mathrm{M}$ small interfering RNA (siRNA; sequence: 5'-CCATTA AGCTTGAGGCAAT-3'; 5'-ATTGCCTCAAGCTTAATG G-3'), or negative control siRNA (sequence: 5'-UUCUCCGAA CGUGUCACGUTT-3'; 5'-ACGUGACACGUUCGGAGA ATT-3'), synthesized by Guangzhou Forevergen Biosciences Co., Ltd., Guangzhou, China and dissolved in $250 \mu \mathrm{l} \mathrm{Gibco}{ }^{\mathrm{TM}}$ Opti-MEM (Gibco; Thermo Fisher Scientific, Inc.). The cells were then incubated with $5 \mu$ l Lipofectamine ${ }^{\circledR} 2000$ transfection reagent (Invitrogen; Thermo Fisher Scientific, Inc.), which was pre-mixed with $250 \mu \mathrm{l} \mathrm{Opti-MEM}$, at $37^{\circ} \mathrm{C}$ for $4 \mathrm{~h}$ in an atmosphere containing $5 \% \mathrm{CO}_{2}$. Finally, cells were cultured in fresh DMEM $48 \mathrm{~h}$ prior to the subsequent assays.

Cell proliferation assay. Proliferation rates of breast cancer cells were determined using the MTS method. A single-cell suspension was prepared by digesting breast cancer cells with trypsin solution, followed by cell counting. Cell density was adjusted to $3 \times 10^{4}$ cells $/ \mathrm{ml}$, and cells were seeded into 96 -well plates (100 $\mu \mathrm{l} /$ well). MTS solution (10 $\mu \mathrm{l}$; cat. no. ab197010; Abcam, Cambridge, UK) was added to the cultured breast cancer cells at a 1:10 ratio, and cells were incubated at $37^{\circ} \mathrm{C}$ for $4 \mathrm{~h}$ in an atmosphere containing $5 \% \mathrm{CO}_{2}$. Finally, cell proliferation rates were measured by detecting the absorbance at $490 \mathrm{~nm}$ using a microplate reader. At least three biological repeats were performed for statistical analysis.

Cell migration and invasion assays. To evaluate breast cancer cell migration, cultured cells were collected by trypsin digestion and centrifugation at $100 \mathrm{x} \mathrm{g}$ for $1 \mathrm{~min}$ at room temperature, diluted in serum-free medium to obtain $1 \times 10^{5}$ cells $/ \mathrm{ml}$, and a $100-\mu 1$ cell suspension was added to the upper chambers of a Transwell system. The lower chambers of the Transwell system were filled with $600 \mu 1$ fresh DMEM. After being cultured under normal conditions for $48 \mathrm{~h}$, migrated cells were fixed with $4 \%$ paraformaldehyde for $15 \mathrm{~min}$, stained with $1 \%$ crystal violet for $10 \mathrm{~min}$ both at room temperature, washed with PBS, and finally observed and counted under an inverted fluorescence microscope (Leica Microsystems GmbH, Wetzlar, Germany). To assess cell invasion, Matrigel matrix (Corning Incorporation, Corning, NY, USA) was mixed with DMEM at a ratio of 1:3, and was added to the Transwell chambers and incubated at $37^{\circ} \mathrm{C}$ for $2 \mathrm{~h}$. Cells were then cultured for $48 \mathrm{~h}$ at $37^{\circ} \mathrm{C}$, fixed with $4 \%$ paraformaldehyde for $15 \mathrm{~min}$, stained with $1 \%$ crystal violet for $10 \mathrm{~min}$, both at room temperature, and finally analyzed under a microscope.

Cell cycle progression analysis. Breast cancer cells ( $80 \%$ confluence) were washed three times with PBS, and a single-cell suspension was generated through trypsin digestion. Subsequently, $\sim 1 \times 10^{6}$ cells were fixed with $70 \%$ ethanol at $-20^{\circ} \mathrm{C}$ overnight. Cells were then collected by centrifugation at $500 \mathrm{x} \mathrm{g}$ for $5 \mathrm{~min}$ and were washed twice with PBS. Cell precipitates were then resuspended in $500 \mathrm{ml}$ Cell Cycle staining buffer (cat. no. F559763; Guangzhou Forevergen Biosciences Co., Ltd.), incubated at $37^{\circ} \mathrm{C}$ for $30 \mathrm{~min}$, and finally detected by flow cytometry (Sysmex Partec $\mathrm{GmbH}$, Görlitz, Germany). Three biological replicates were performed for statistical analysis.

Cell apoptosis assay. Cell apoptosis was determined using the Annexin V-allophycocyanin/7-aminoactinomycin D (7-AAD) Apoptosis Detection kit (cat. no. 40309ES20; Yeasen, Shanghai, China), according to the manufacturer's protocol. Breast cancer cells were collected by digestion with EDTA-free trypsin solution and were centrifuged at $800 \mathrm{x} g$ for $5 \mathrm{~min}$. Cell precipitates were then resuspended in $1 \mathrm{X}$ binding buffer, and cell density was adjusted to $1 \times 10^{6}$ cells $/ \mathrm{ml}$. Finally, $100 \mu \mathrm{l}$ cells were incubated with $5 \mu \mathrm{l}$ Annexin $\mathrm{V}$ and $5 \mu 1$ 7-AAD in the dark for $15 \mathrm{~min}$ at room temperature, incubated with $400 \mu \mathrm{l}$ binding buffer for $1 \mathrm{~h}$, and analyzed by flow cytometry (Sysmex Partec GmbH). Data from three biological repeats were used for statistical analysis.

Western blotting. Protein was extracted using RIPA Lysis Buffer (Beyotime Institute of Biotechnology, Haimen, China). The concentration was determined using the Bicinchoninic Acid Kit for Protein Determination (Sigma-Aldrich; Merck KGaA). Total proteins were extracted from breast tissues and breast cancer cell lines for immunoblotting. Briefly, $35 \mu \mathrm{g}$ total proteins were boiled at $100^{\circ} \mathrm{C}$ for $5 \mathrm{~min}$, loaded and separated by $12 \%$ SDS-PAGE, and electroblotted onto polyvinylidene fluoride (PVDF) membranes (EMD Millipore, Billerica, MA, USA). PVDF membranes were then blocked with $5 \%$ lipid-free milk solution for $1 \mathrm{~h}$ at room temperature, and incubated with primary antibodies against phosphorylated (p)-checkpoint kinase 2 p-CHK2 (cat. no. ab59408; 1:1,000), CHK2 (cat. no. ab47433; 1:1,000), p-protein kinase B p-AKT (cat. no. ab38449; 1:500) and AKT (cat. no. ab179463; 1:500; all Abcam) at room temperature for $1 \mathrm{~h}$. The membranes were then washed three times with PBS, incubated with horseradish peroxidase-conjugated secondary antibodies (cat. no. ab6940; 1:10,000; Abcam) at room temperature for $1 \mathrm{~h}$, and finally detected with enhanced chemiluminescence solutions (Thermo Fisher Scientific, Inc., Waltham, MA, USA). GAPDH (cat. no. ab181602; 1:10,000; Abcam) was used as an internal standard, and three biological repeats were performed. Signals were densitometrically assessed using Quantity One ${ }^{\circledR}$ software version 4.5 (Bio Rad Laboratories, Inc., Hercules, CA, USA).

Statistical analysis. Data were presented as mean \pm standard error of the mean. The present findings were statistically analyzed using SPSS 18.0 software package (SPSS, Inc., Chicago, IL, USA) via Student's t-test or one way analysis of variance, least significant difference test was used to analyze the differences among more than two groups. $\mathrm{P}<0.05$ was considered to indicate a statistically significant difference.

\section{Results}

PRNCR1-2 expression is elevated in breast cancer. To identify a novel lncRNA associated with breast cancer pathogenesis, the expression levels of PRNCR1-2 were analyzed 

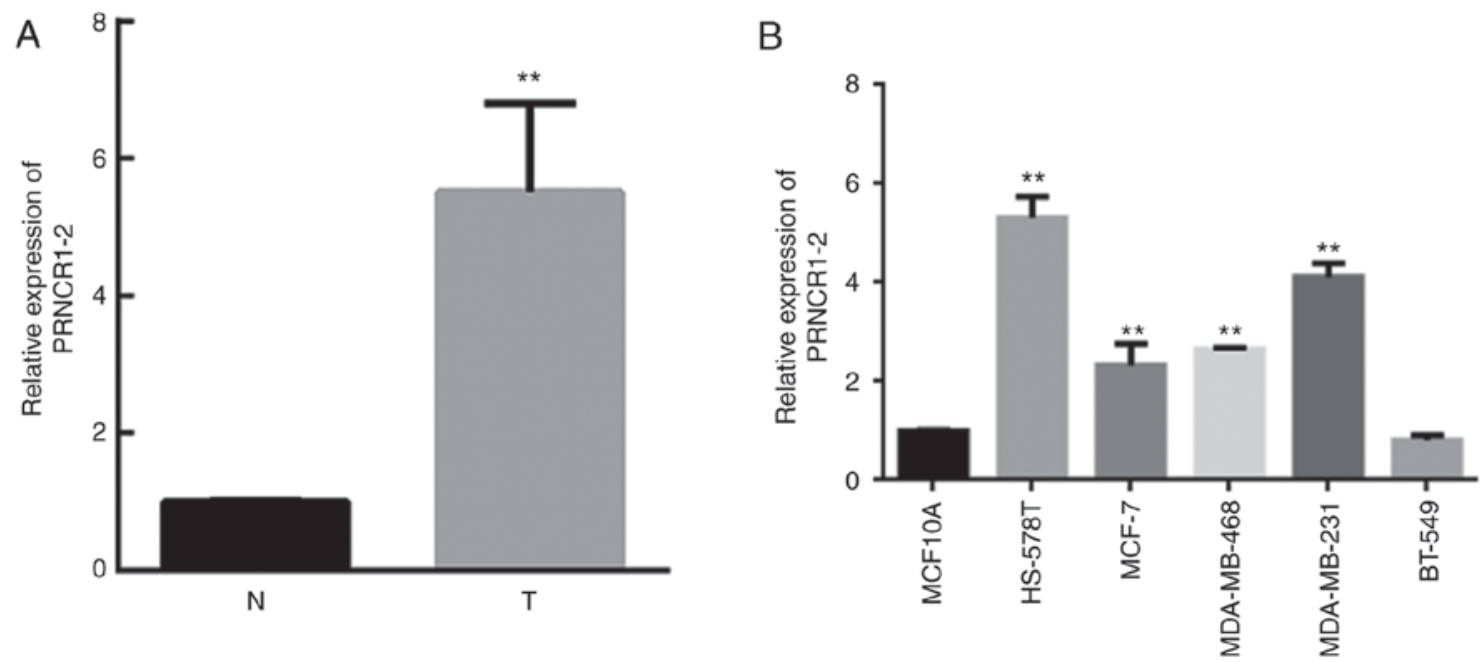

Figure 1. PRNCR1-2 expression in breast cancer. (A) PRNCR1-2 expression in breast cancer tissues and paired adjacent normal tissues. (B) PRNCR1-2 expression in MCF10A and five breast cancer cell lines. The relative expression levels of PRNCR1-2 were determined by reverse transcription-quantitative polymerase chain reaction. ${ }^{* *} \mathrm{P}<0.01$ vs. $\mathrm{N}$ or MCF10A. N, normal adjacent tissues; PRNCR1-2, prostate cancer-associated non-coding RNA 1-2; T, tumor tissues.
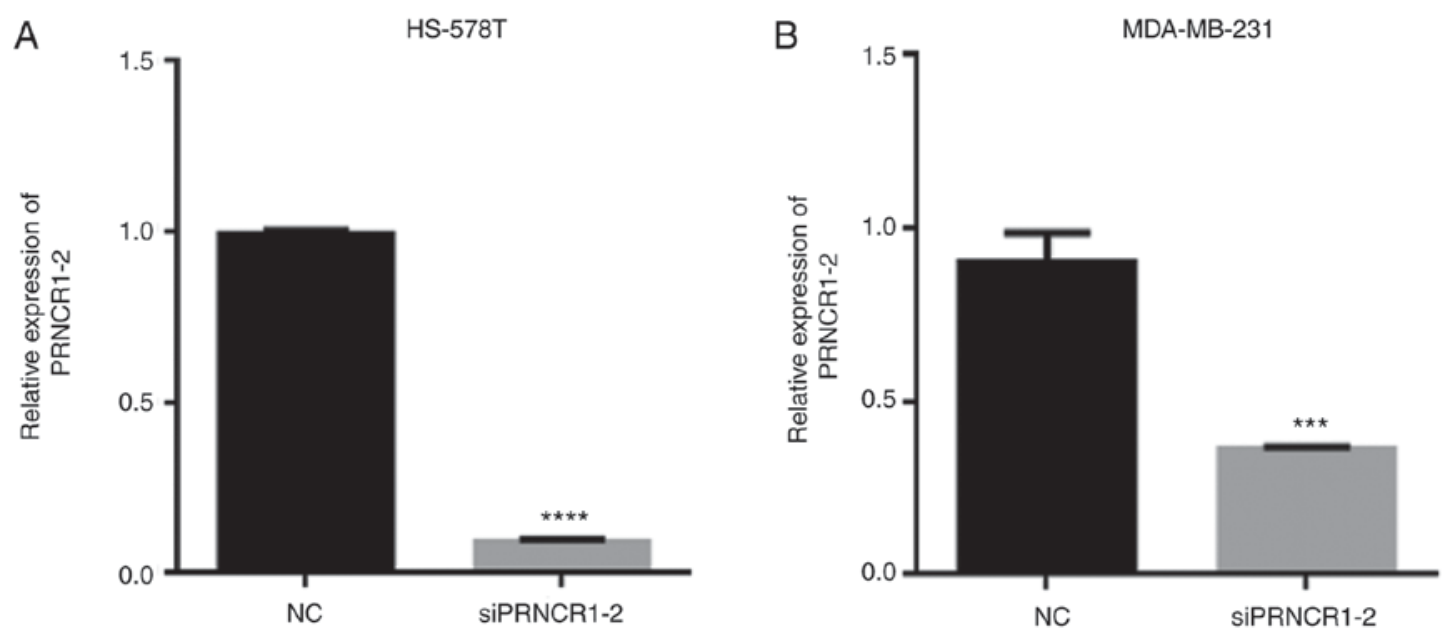

Figure 2. Depletion of PRNR1-2 expression in breast cancer cells. (A) Expression of PRNCR1-2 in HS-587T breast cancer cells transfected with siPRNCR1-2. (B) Expression of PRNCR1-2 in MDA-MB-231 breast cancer cells transfected with siPRNCR1-2. Reverse transcription-quantitative polymerase chain reaction was conducted for PRNCR1-2 expression in HS-587T and MDA-MB-231 cells. ${ }^{* * *} \mathrm{P}<0.001 ;{ }^{* * * *} \mathrm{P}<0.0001$. NC, negative control; PRNCR1-2, prostate cancer-associated non-coding RNA 1; si, small interfering RNA.

in 20 pairs of breast cancer and adjacent normal tissues (Fig. 1A). Compared with the normal tissues, the expression levels of PRNCR1-2 were significantly elevated in breast cancer tissues $(\mathrm{P}<0.05$; Fig. $1 \mathrm{~A})$. For further validation, PRNCR1-2 expression was confirmed in five breast cancer cell lines and a mammary epithelial cell line; PRNCR1-2 were successfully overexpressed in the cancer cell lines HS-587T, MCF-7, MDA-MB-468 and MDA-MB-231 compared with the mammary epithelial cell line MCF10A, but failed to overexpress in the cancer cell line BT-549 (Fig. 1B). The upregulation of PRNCR1-2 expression in breast cancer tissues suggested that PRNCR1-2 may have pathogenic roles in breast cancer. Due to the high expression level of PRNCR1-2 in HS-587T and MDA-MB-231 cells, these two cell lines were chosen for the following studies.

PRNCR1-2 depletion in HS-587T and MDA-MB-231 cells. To investigate the potential roles of the lncRNA
PRNCR1-2 in breast cancer pathogenesis, HS-587T and MDA-MB-231 breast cancer cells were transfected with a specific siRNA against PRNCR1-2. It was revealed that transfection with siRNA-PRNCR1-2 markedly reduced PRNCR1-2 expression in HS-587T and MDA-MB-231 cells, compared with in the negative control group (Fig. 2A and B). siRNA-PRNCR1-2-transfected HS-587T and MDA-MB-231 cells were used for subsequent functional assays.

PRNCR1-2 regulates breast cancer cell proliferation, migration and invasion. To explore the possible roles of PRNCR1-2 in breast cancer initiation and progression, the proliferation, migration, invasion, cell cycle progression and apoptosis of siRNA-PRNCR1-2-transfected HS-587T and MDA-MB-231 cells were analyzed. The results of the MTS assay revealed that the proliferation rates of siRNA-PRNCR1-2-transfected HS-587T cells were markedly downregulated compared with in the negative control 
A

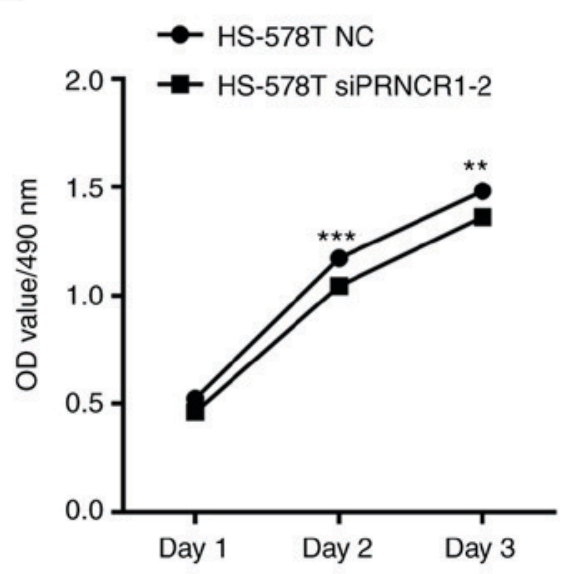

C

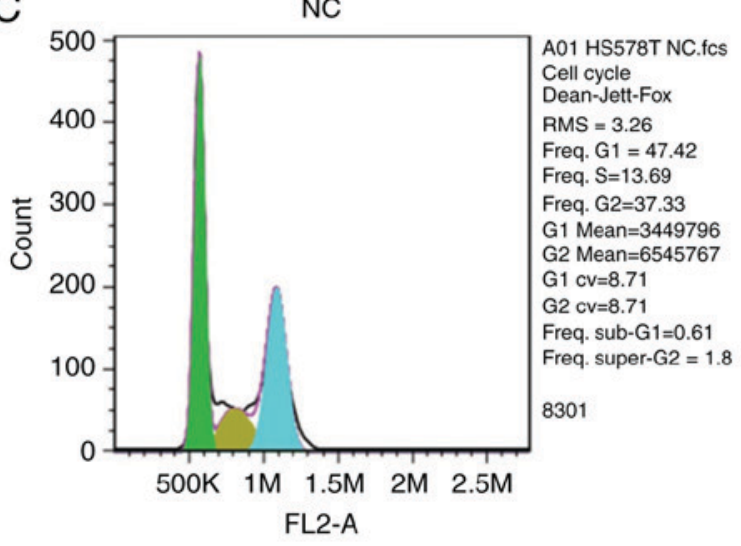

D

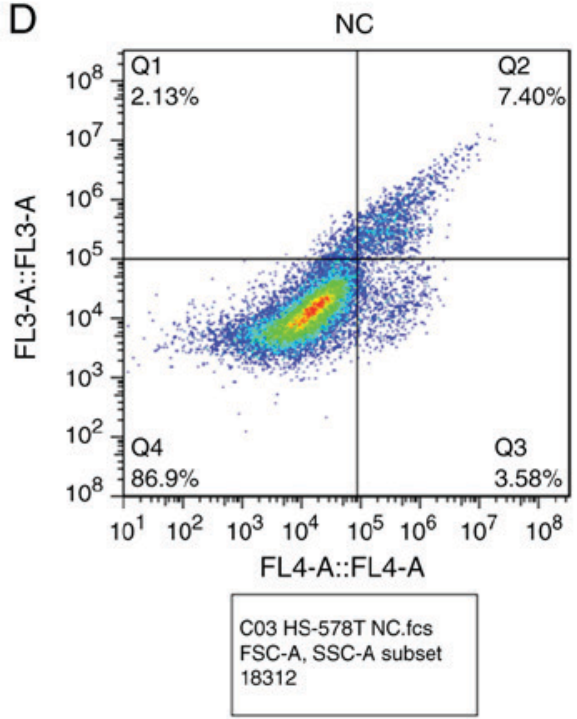

B
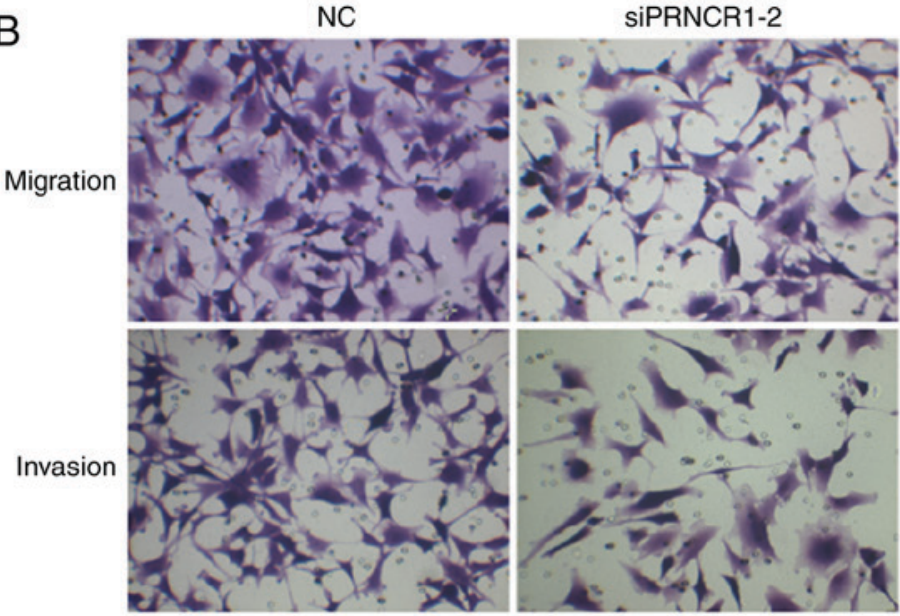
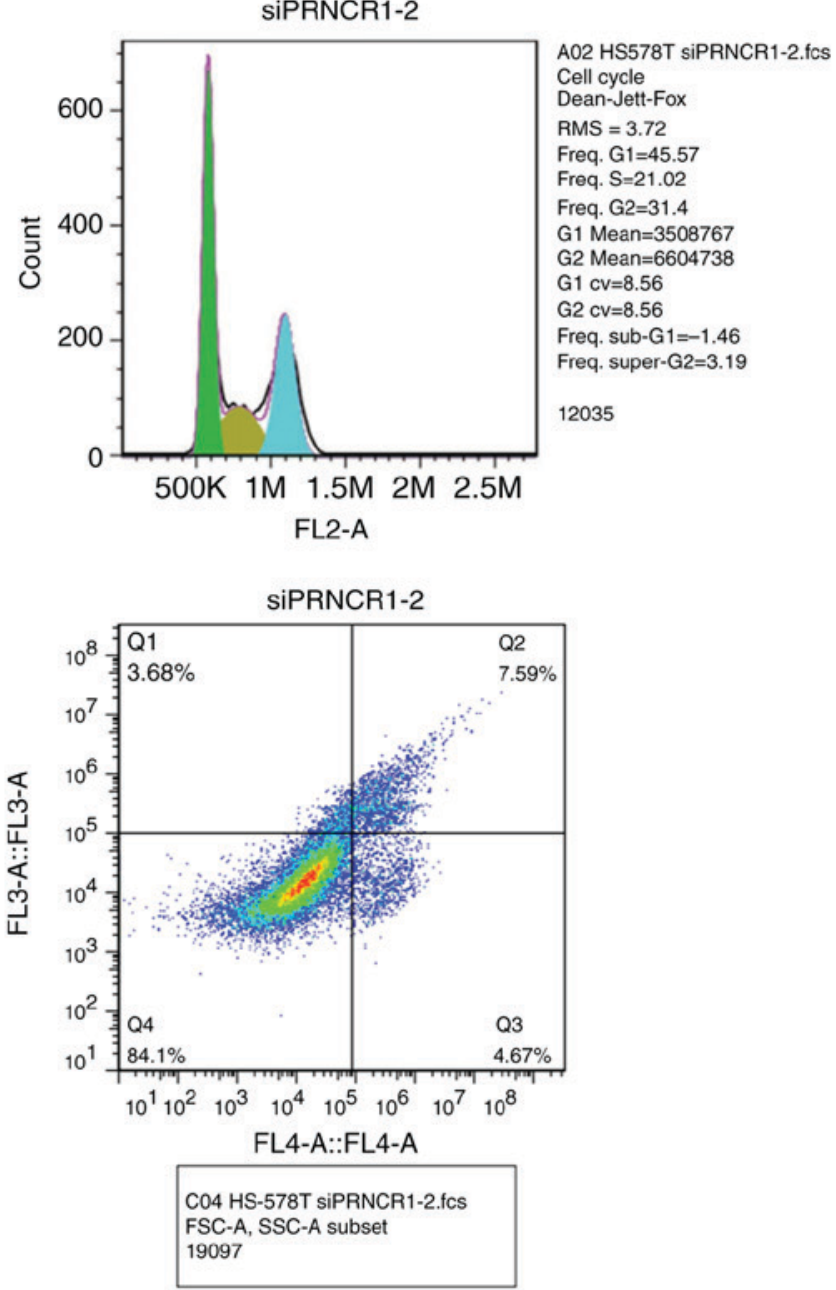

Figure 3. Cellular properties of HS-587T cells with PRNCR1-2 knockdown. (A) Proliferation of HS-587T cells with PRNCR1-2 knockdown. Cell proliferation was assessed by MTS assay. (B) Migration and invasion of HS-587T cells transfected with siPRNCR1-2. Transwell assays were used for analysis of cell migration and invasion. (C) Cell cycle progression of PRNCR1-2-depleted HS-587T cells. (D) Apoptotic cell ratios of PRNCR1-2-depleted HS-587T cells. Cell cycle progression and apoptosis were analyzed by flow cytometry. ${ }^{* *} \mathrm{P}<0.01 ;{ }^{* * *} \mathrm{P}<0.001$. NC, negative control; PRNCR1-2, prostate cancer-associated non-coding RNA 1-2; si, small interfering RNA.

group (Fig. 3A), thus indicating the ability of PRNCR1-2 to modulate breast cancer cell proliferation. Furthermore, the migration and invasion of PRNCR1-2-depleted HS-587T cells were analyzed using Transwell assays; the migration and invasion of HS-587T cells were markedly suppressed by transfection with siRNAs targeting PRNCR1-2 (Fig. 3B). Consistent with PRNCR1-2 depletion-induced alterations in proliferation, the cell cycle progression of siRNA-PRNCR1-2-transfected HS-587T cells was markedly altered, with more cells arrested in $\mathrm{S}$ phase, thus indicating 
A

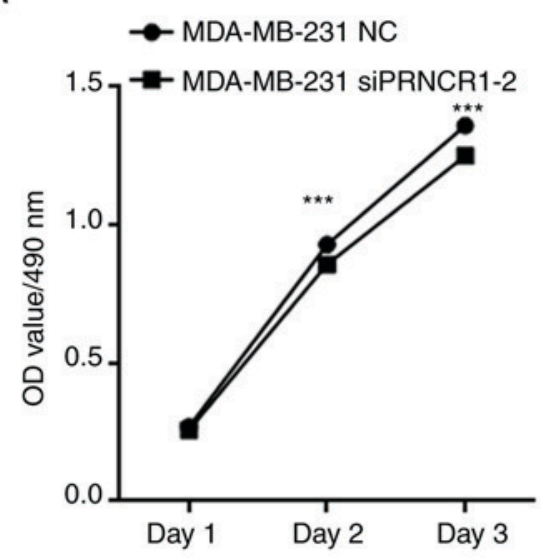

B

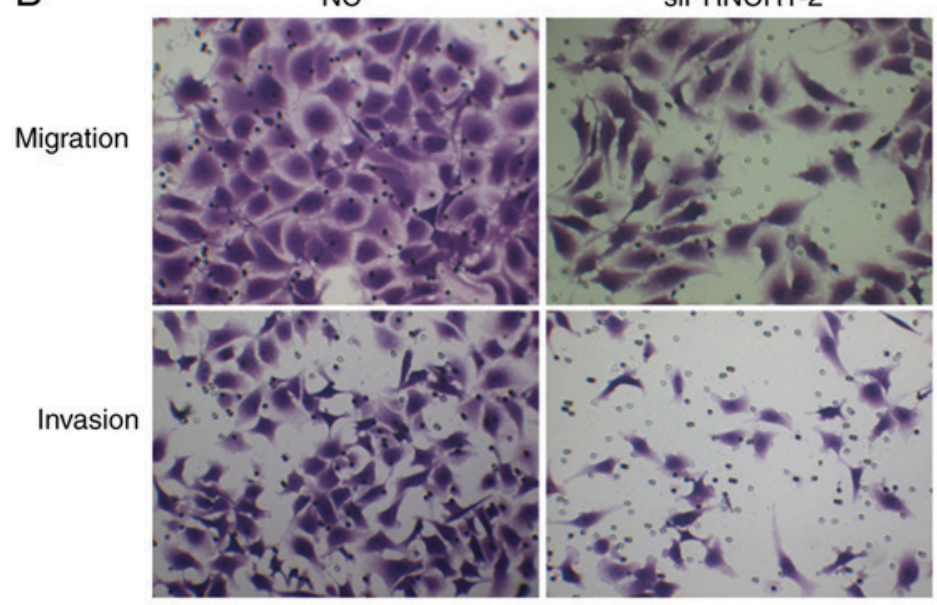

C

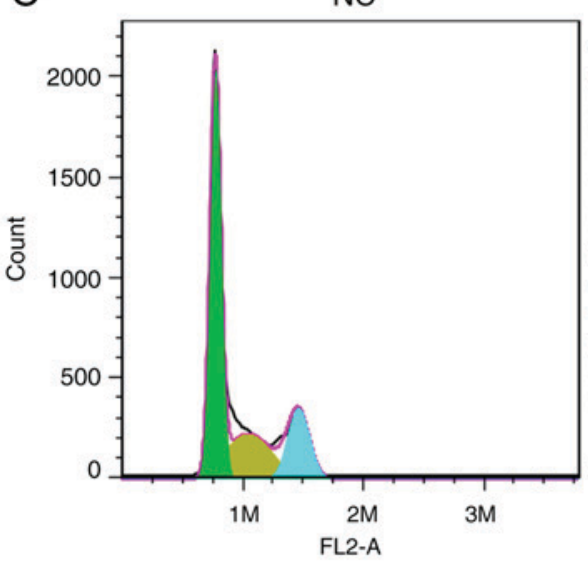

NC

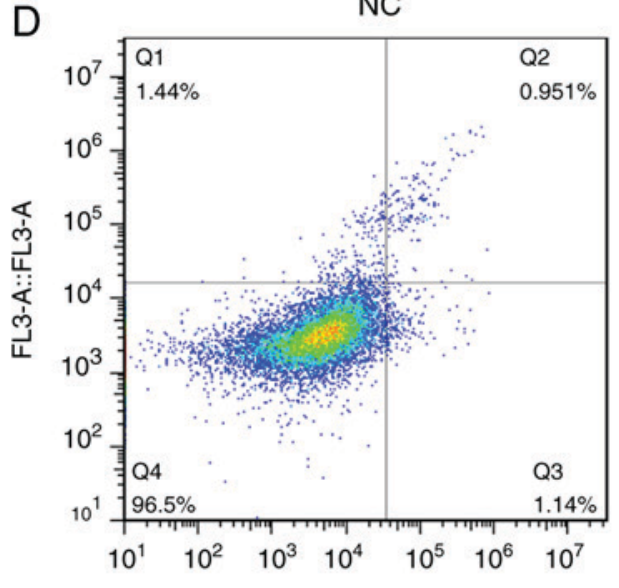

FL4-A::FL4-A

C01 MDA231NC.fCS

FSC-A, SSC-A subse

15289
C01 MDA231 NC.fCs Cell cycle Dean-Jett-Fox

RMS $=10.45$

Freq. $\mathrm{G} 1=57.99$

Freq. $\mathrm{S}=23.9$

Freq. G2 $=19.71$

G1 Mean $=3449796$

G2 Mean $=6486797$

$\mathrm{G} 1 \mathrm{cv}=8.29$

$\mathrm{G} 2 \mathrm{cv}=8.7$

Freq. sub-G1 $=-1.26$

Freq. super-G2 $=1.15$

27358
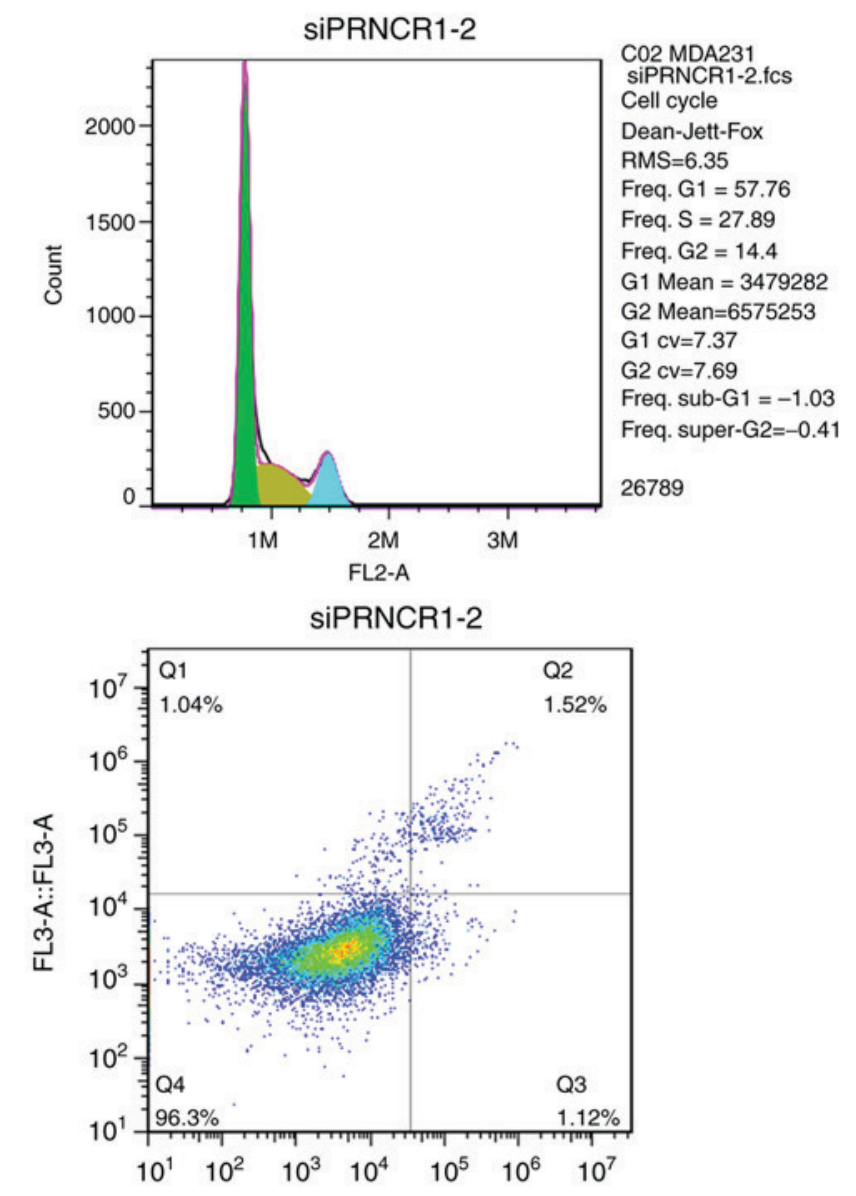

FL4-A::FL4-A

C01 MDA231 siPRNCR1-2.fcs

FSC-A, SSC-A subset

15243

Figure 4. Cellular properties of MDA-MB-231 cells with PRNCR1-2 knockdown. (A) Proliferation of MDA-MB-231 cells with PRNCR1-2 knockdown. Cell proliferation was assessed by MTS assay. (B) Migration and invasion of MDA-MB-231 cells transfected with siPRNCR1-2. Transwell assays were used for analysis of cell migration and invasion. (C) Cell cycle progression of PRNCR1-2-depleted MDA-MB-231 cells. (D) Apoptotic cell ratios of PRNCR1-2-depleted MDA-MB-231 cells. Cell cycle progression and apoptosis were analyzed by flow cytometry. ${ }^{* * *} \mathrm{P}<0.001$. NC, negative control; PRNCR1-2, prostate cancer-associated non-coding RNA 1-2; si, small interfering RNA.

the involvement of PRNCR1-2 in promoting $\mathrm{S} / \mathrm{G}_{2}$ transition of breast cancer cells (Fig. 3C). The proportion of apoptotic cells in the siRNA-PRNCR1-2 group was also analyzed by flow cytometry; however, no marked alterations in cell apoptosis were observed in siRNA-PRNCR1-2-transfected HS-587T cells (Fig. 3D). The aforementioned experiments 


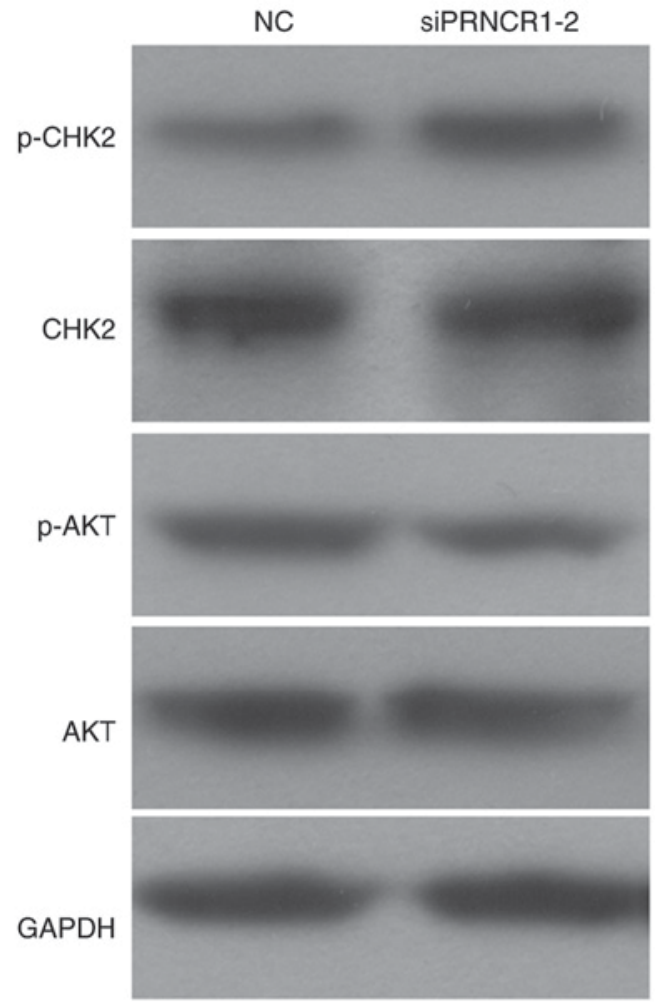

HS-578T
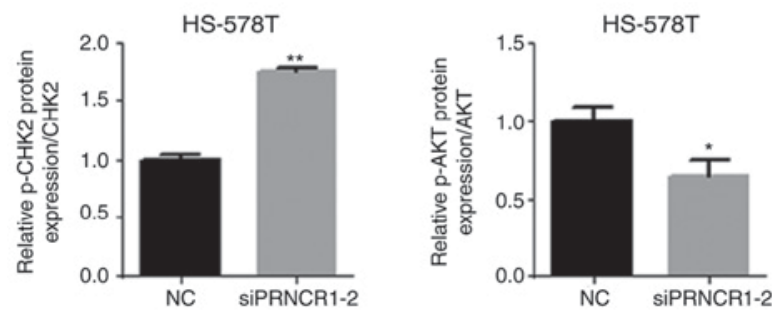

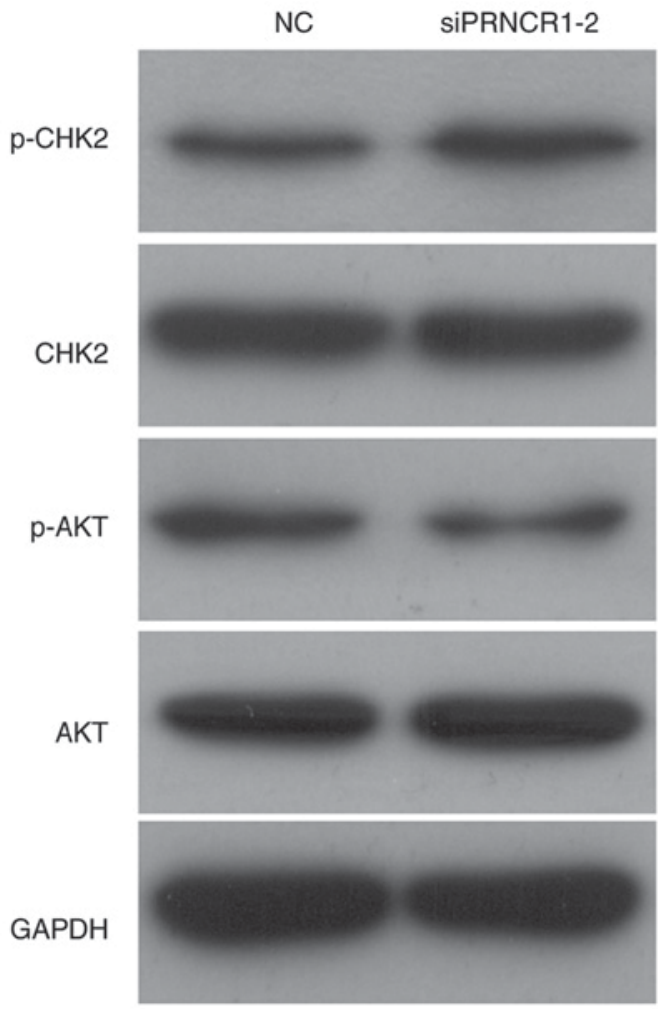

MDA-MB-231
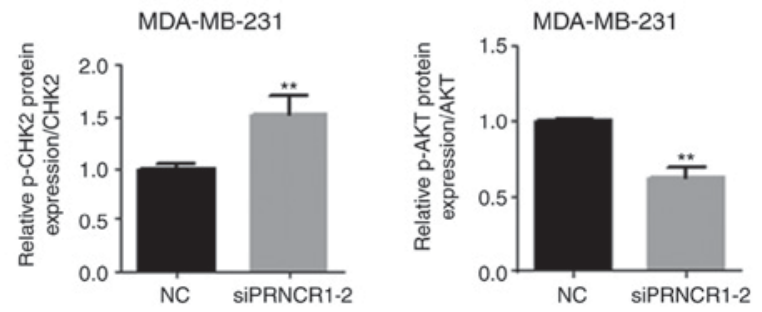

Figure 5. Signaling pathways regulated by PRNCR1-2 in breast cancer cells. The abundance and phosphorylation of key components associated with cell proliferation, migration, invasion, apoptosis and cell cycle progression in HS-587T and MDA-MB-231 cells transfected with siPRNCR1-2 were determined by western blotting. GAPDH was used as an internal standard. "P $<0.05$; ${ }^{* *} \mathrm{P}<0.01$. AKT, protein kinase $\mathrm{B}$; CHK2, checkpoint kinase 2; NC, negative control; $\mathrm{p}$, phosphorylated; PRNCR1-2, prostate cancer-associated non-coding RNA 1-2; si, small interfering RNA.

were repeated in MDA-MB-231 cells and similar results were obtained, as shown in Fig. 4. Taken together, these cellular assays suggested that PRNCR1-2 may be involved in breast cancer progression by promoting cell proliferation, migration and invasion, and by modulating cell cycle progression, but not via modulation of breast cell apoptosis.

Signaling in HS-587T and MDA-MB-231 cells is modulated by PRNCR1-2 depletion. For more insights into PRNCR1-2-induced regulation of cell functions during breast cancer progression, key signaling pathway components associated with cell proliferation, migration, invasion, cell cycle progression and apoptosis were detected in HS-587T and MDA-MB-231 cells transfected with siRNA-PRNCR1-2 by western blotting. The results demonstrated that the protein expression levels of p-CHK2 were significantly elevated in siRNA-PRNCR1-2-transfected HS-587T and MDA-MB-231 cells (Fig. 5). Furthermore, depletion of PRNCR1-2 expression using specific siRNA resulted into significant inhibition of p-AKT in HS-587T and MDA-MB-231 cells; however, PRNCR1-2 depletion had no effect on the abundance of total CHK2 and AKT proteins (Fig. 5). The selectively altered expression of key signaling components suggested that regulation of breast cancer cell proliferation, migration, invasion and cell cycle progression by PRNCR1-2 may be at least partially mediated by modulating phosphorylation of CHK2 and AKT.

\section{Discussion}

Non-coding components that account for large volumes of the human genome have long been predicted to have important roles in various physiological and pathological processes (19). Various types of ncRNAs, including microRNAs, siRNAs, Piwi-interacting RNAs, small nucleolar RNAs and lncRNAs critically contribute to cancer initiation and progression, much more than previously expected $(8,10-12,20)$. Increasing evidence has suggested that lncRNAs may be involved in tumor pathogenesis, thus promoting trials of their application in breast cancer diagnosis and prognosis. The lncRNA H19 is characterized as a highly expressed biomarker in ER-positive MCF-7 breast cancer cells, which is involved in breast cell 
survival and estrogen-induced cell proliferation during breast cancer development (21). In addition, its diagnostic value as a novel biomarker for breast cancer has been reported, due to its detectable expression in the urine and serum of patients with breast cancer $(22,23)$. Accumulating numbers of such ncRNAs as candidate targets for cancer diagnosis and treatment highlight the importance of further characterization of novel ncRNAs in breast cancer pathogenesis.

Aiming to provide a novel candidate for breast cancer diagnosis and treatment, the present study analyzed the expression levels of the lncRNA PRNCR1-2 in cancer tissues collected from patients with breast cancer. PRNCR1-2 expression was significantly increased in cancer tissues, thus suggesting its potential association with breast cancer development. Subsequently, a knockdown assay was conducted to deplete PRNCR1-2 expression in breast cancer cells. The marked alterations in breast cancer cell proliferation, migration, invasion and cell cycle progression following suppression of PRNCR1-2 expression indicated that PRNCR1-2 may be considered a novel non-coding regulator in breast cancer pathogenesis. Significantly altered AKT and CHK2 phosphorylation further validated the critical roles served by PRNCR1-2 in breast cancer cells. In accordance with previous findings regarding lncRNAs in breast cancer (8), the present results revealed the cellular functions of PRNCR1-2 in breast cancer cells, which may be applied as a biomarker for breast cancer diagnosis and therapy.

The molecular mechanisms by which PRNCR1-2 promotes breastcancercell proliferation, migration and cell cycle progression deserve further investigations. Previous studies reported that interactions of IncRNAs with proteins or RNA partners may be important for the functioning of lncRNA molecules $(10,24)$. A recent study also demonstrated that lncRNAs can bind with Janus kinase 2 (JAK2), thus promoting JAK2 activation and signal transducer and activation of transcription 3 phosphorylation, finally mediating breast cancer brain metastases (25). Therefore, it is reasonable to speculate that PRNCR1-2 may also carry out its role in breast cancer cell proliferation and migration via its association with key protein components of these cellular processes. The large-scale identification of proteins or RNA partners interacting with PRNCR1-2 during breast cancer development may provide novel perspectives on pathogenic roles of non-coding molecules in breast cancer, as well as other types of human cancer. It is also possible that PRNCR1-2 may be directly involved in the post-translational modifications of key signaling proteins. Metastasis associated in lung adenocarcinoma transcript 1 (MALAT1) interacts with both serine and arginine-rich splicing factor 1 (SRSF1) and SRSF protein kinase 1. In colorectal cancer cells, the lncRNA MALAT1 regulates cancer cell proliferation and migration by promoting phosphorylation of SRSF1 (26). In the present study, it was revealed that depletion of PRNCR1-2 markedly altered the phosphorylation of AKT and CHK2, whereas total AKT and CHK2 protein levels were not affected. CHK2 acts as an important regulator of cell cycle progression and proliferation $(27,28)$, and CHK2 signaling is activated by phosphorylation of itself and downstream substrates (29). AKT is another key regulator of tumor cell proliferation, cell cycle progression, migration and invasion (30-32), which is also activated by its phosphorylation (33). These observations suggested that PRNCR1-2 may modulate the phosphorylation of CHK2 and AKT by interacting with a specific kinase or phosphatase. The role of PRNCR1-2 in CHK2 and AKT phosphorylation and regulation requires further investigation.

In conclusion, this study reported that the lncRNA PRNCR1-2 is highly expressed in breast cancer tissues, and depletion of PRNCR1-2 in breast cancer cells results in the suppression of cell proliferation, migration, invasion and cell cycle progression. These findings indicated that PRNCR1-2 may be explored as a breast cancer biomarker for the development of novel diagnostic or therapeutic methods for patients with breast cancer.

\section{Acknowledgements}

The present study was supported by Guangzhou Forevergen Company. The authors would also like to thank Dr Kewei Li of Foshan First People's Hospital for the specimen collection.

\section{Funding}

The present study was supported by grants from the Specialized Research Fund for the Technology Innovation of Foshan City (grant no. 2014AG10003) and the Research Fund for the Guangdong Province Health Bureau (grant no. A2017464).

\section{Availability of data and materials}

The datasets used and/or analyzed during the current study are available from the corresponding author on reasonable request.

\section{Authors' contributions}

DP and QH conceived and designed the study, and drafted and critically revised the manuscript. XL and YXL performed the experiments and analyzed the data. HD, SC, YDL, LL, FPe and FPa participated in study design, study implementation and manuscript revision. All authors read and approved the final manuscript.

\section{Ethics approval and consent to participate}

The experimental procedures were approved by the Ethics Committee of Foshan First People's Hospital, and written informed consent was obtained from each participant prior to surgery.

\section{Patient consent for publication}

Not applicable.

\section{Competing interests}

The authors declare that they have no competing interests.

\section{References}

1. Siegel RL, Miller KD and Jemal A: Cancer statistics, 2018. CA Cancer J Clin 68: 7-30, 2018.

2. Desantis C, Ma J, Bryan L and Jemal A: Breast cancer statistics, 2013. CA Cancer J Clin 64: 52-62, 2014. 
3. Bombonati A and Sgroi DC: The molecular pathology of breast cancer progression. J Pathol 223: 307-317, 2011.

4. Lopez-Garcia MA, Geyer FC, Lacroix-Triki M, Marchió C and Reis-Filho JS: Breast cancer precursors revisited: Molecular features and progression pathways. Histopathology 57: 171-192, 2010.

5. Byler S, Goldgar S, Heerboth S, Leary M, Housman G, Moulton K and Sarkar S: Genetic and epigenetic aspects of breast cancer progression and therapy. Anticancer Res 34: 1071-1077, 2014.

6. Welch DR and Wei LL: Genetic and epigenetic regulation of human breast cancer progression and metastasis. Endocrine-Related Cancer 5: 155-197, 1998.

7. Mercer TR and Mattick JS: Structure and function of long noncoding RNAs in epigenetic regulation. Nat Struct Mol Biol 20: 300-307, 2013.

8. Lo PK, Wolfson B, Zhou X, Duru N, Gernapudi R and Zhou Q: Noncoding RNAs in breast cancer. Brief Funct Genomics 15 : 200-221, 2016.

9. Cao J: The functional role of long non-coding RNAs and epigenetics. Biol Proced Online 16: 11, 2014.

10. Lin C and Yang L: Long noncoding RNA in cancer: Wiring signaling circuitry. Trends Cell Biol 28: 287-301, 2018.

11. Slaby O, Laga R and Sedlacek O: Therapeutic targeting of non-coding RNAs in cancer. Biochem J 474: 4219-4251, 2017.

12. Huarte M: The emerging role of lncRNAs in cancer. Nature Medicine 21: 1253-1261, 2015.

13. Sørensen KP, Thomassen M, Tan Q, Bak M, Cold S, Burton M, Larsen MJ and Kruse TA: Long non-coding RNA HOTAIR is an independent prognostic marker of metastasis in estrogen receptor-positive primary breast cancer. Breast Cancer Res Treat 142: 529-536, 2013

14. Huang J, Zhou N, Watabe K, Lu Z, Wu F, Xu M and Mo YY: Long non-coding RNA UCA1 promotes breast tumor growth by suppression of p27 (Kip1). Cell Death Dis 5: e1008, 2014.

15. Prensner JR, Sahu A, Iyer MK, Malik R, Chandler B, Asangani IA, Poliakov A, Vergara IA, Alshalalfa M, Jenkins RB, et al: The IncRNAs PCGEM1 and PRNCR1 are not implicated in castration resistant prostate cancer. Oncotarget 5: 1434-1438, 2014.

16. Yang L, Qiu M, Xu Y, Wang J, Zheng Y, Li M, Xu L and Yin R Upregulation of long non-coding RNA PRNCR1 in colorectal cancer promotes cell proliferation and cell cycle progression. Oncol Rep 35: 318-324, 2016.

17. Sattarifard H, Hashemi M, Hassanzarei S, Narouie B and Bahari G: Association between genetic polymorphisms of long non-coding RNA PRNCR1 and prostate cancer risk in a sample of the Iranian population. Mol Clin Oncol 7: 1152-1158, 2017.

18. Livak KJ and Schmittgen TD: Analysis of relative gene expression data using real-time quantitative PCR and the 2(-Delta Delta C(T)) method. Methods 25: 402-408, 2001

19. Esteller M: Non-coding RNAs in human disease. Nature Reviews Genetics 12: 861-874, 2011.

20. Jing H, Markowitz GJ and Wang X: Noncoding RNAs regulating cancer signaling network. Adv Exp Med Biol 927: 297-315, 2016.
21. Sun H, Wang G, Peng Y,Zeng Y,Zhu QN, Li TL, Cai JQ,Zhou HH and Zhu YS: H19 lncRNA mediates $17 \beta$-estradiol-induced cell proliferation in MCF-7 breast cancer cells. Oncol Rep 33: 3045-3052, 2015

22. Zhang K, Luo Z, Zhang Y, Zhang L, Wu L, Liu L, Yang J, Song X and Liu J: Circulating lncRNA H19 in plasma as a novel biomarker for breast cancer. Cancer Biomark 17: 187-194, 2016.

23. Zhang K, Zhang Y, Luo Z, Lichun WU, Zhang L and Liu J: Diagnostic value of urinary lncRNA H19 for breast cancer. Shandong Med J 56: 42-44, 2016.

24. Lin A, Hu Q, Li C, Xing Z, Ma G, Wang C, Li J, Ye Y, Yao J, Liang K, et al: The LINK-AlncRNA interacts with PtdIns $(3,4,5)$ $\mathrm{P}_{3}$ to hyperactivate AKT and confer resistance to AKT inhibitors. Nature Cell Biol 19: 238-251, 2017.

25. Wang S, Liang K, Hu Q, Li P, Song J, Yang Y, Yao J, Mangala LS, Li C, Yang W, et al: JAK2-binding long noncoding RNA promotes breast cancer brain metastasis. J Clin Invest 127: 4498-4515, 2017

26. Hu ZY, Wang XY, Guo WB, Xie LY, Huang YQ, Liu YP, Xiao LW, Li SN, Zhu HF, Li ZG and Kan H: Long non-coding RNA MALAT1 increases AKAP-9 expression by promoting SRPK1-catalyzed SRSF1 phosphorylation in colorectal cancer cells. Oncotarget 7: 11733-11743, 2016.

27. Ingvarsson S, Sigbjornsdottir BI, Chen H, Hafsteinsdottir SH, Ragnarsson G, Barkardottir RB, Arason A, Egilsson V and Bergthorsson JT: Mutation analysis of the CHK2 gene in breast carcinoma and other cancers. Breast Cancer Res 4: 1-6, 2002.

28. Sullivan A, Yuille M, Repellin C, Reddy A, Reelfs O, Bell A, Dunne B, Gusterson BA, Osin P, Farrell PJ, et al: Concomitant inactivation of p53 and Chk2 in breast cancer. Oncogene 21: 1316-1324, 2002

29. Xu X, Tsvetkov LM and Stern DF: Chk2 activation and phosphorylation-dependent oligomerization. Mol Cell Biol 22: 4419-4432, 2002.

30. Hartmann W, Digonsöntgerath B, Koch A, Waha A, Endl E, Dani I, Denkhaus D, Goodyer CG, Sörensen N, Wiestler OD and Pietsch T: Phosphatidylinositol 3'-kinase/AKT signaling is activated in medulloblastoma cell proliferation and is associated with reduced expression of PTEN. Clin Cancer Res 12: 3019-3027, 2006.

31. Kim D, Kim S, Koh H, Yoon SO, Chung AS, Cho KS and Chung J: Akt/PKB promotes cancer cell invasion via increased motility and metalloproteinase production. FASEB J 15: 1953-1962, 2001.

32. Chang F, Lee JT, Navolanic PM, Steelman LS, Shelton JG, Blalock WL, Franklin RA and McCubrey JA: Involvement of PI3K/Akt pathway in cell cycle progression, apoptosis, and neoplastic transformation: A target for cancer chemotherapy. Leukemia 17: 590-603, 2003

33. Jacinto E, Facchinetti V, Liu D, Soto N, Wei S, Jung SY, Huang Q, Qin J and Su B: SIN1/MIP1 maintains rictor-mTOR complex integrity and regulates Akt phosphorylation and substrate specificity. Cell 127: 125-137, 2006. 